\title{
Relação entre o perímetro escrotal e parâmetros da qualidade do sêmen em machos da raça Guzerá, da puberdade até os 36 meses de idade
}

\author{
Relationship between scrotal circumference and \\ parameters of semen quality in male Guzerat bulls \\ from puberty to 36 months of age raised pasture in the
} savanah

Fecha de recepción: 22 de diciembre de 2015

Fecha de aceptación: 14 de marzo de 2016
Jair Pérez-Osorio ${ }^{1}$

José Alberto Cardona-Álvarez ${ }^{2}$

Víctor Enrique Gómez-León ${ }^{3}$

Rafael José Otero-Arroyo ${ }^{4}$

\begin{abstract}
Resumo
1 Ph. D. Universidad de la Salle (Bogotá D.C., Colombia). jairperez@unisalle.edu.co.

2 Ph. D. Universidad de Córdoba (Córdoba - Montería, Colombia).

3 M. Sc. Universidad de Córdoba (Córdoba - Montería, Colombia).

4 Ph. D. Universidad de Córdoba (Córdoba - Montería, Colombia).
\end{abstract}

O objetivo deste estudo foi verificar a associação entre o tamanho do perímetro escrotal e as características físicas e morfológicas dos ejaculados em 191 touros da raça guzerá. Os touros foram divididos por faixas etárias dos 12 aos 36 meses de idade. As mensurações de perímetro escrotal e medidas testiculares foram obtidas com ajuda de paquímetro e fita métrica de acordo à metodologia do Colégio Brasileiro de Reprodução Animal. Os animais que apresentaram perímetro escrotal $\geq 19 \mathrm{~cm}$ foram submetidos à coleta de sêmen utilizando-se estímulo eletro-ejaculatório. Foram avaliadas as características espermáticas dos ejaculados como motilidade, vigor e também realizadas avaliações de concentração espermática e a presença de células arredondadas. A correlação do perímetro escrotal com a motilidade espermática por faixa etária foi positiva $r=0.94$; $(P<0,005)$. Neste estudo foi observado que a presenças de espermatozoides com gota citoplasmática proximal, células arredondadas no ejaculado e defeitos de cabeça dos espermatozoides são as características de avaliação objetiva que mais poderiam ser utilizados para seleção de precocidade da produção espermática em machos bovinos. Pode ser concluído que a normalidade da produção espermática na raça Guzerá considerando conjuntamente todos os parâmetros representativos 
de maturidade do processo da produção espermática foi atingida com idade acima de 28 meses e perímetro escrotal acima de $30 \mathrm{~cm}$.

Palavras chave: características seminais; Guzerá; morfologia espermática; touros.

\begin{abstract}
The aim of this study was to investigate the association between the size of the scrotal circumference and physical characteristics of semen and sperm morphology, in 191 bulls of Guzerat breed. The bulls were divided by age in six groups from 12 to 36 months of age. Scrotal circumference and testicular measures were obtained by caliper and tape measure, according to the Brazilian College of Animal Reproduction' methodology. The animals with scrotal circumference $\geq 19 \mathrm{~cm}$, were subjected to semen collection using ejaculation by electro-stimulation. The evaluated semen characteristics were sperm motility and vigor, also assessments of sperm concentration and the presence of rounded cells. The correlation between scrotal circumference with sperm motility by age group, was positive $(r=0.94, P<0.005)$.
\end{abstract}

The present study demonstrated that the presence of sperm with proximal cytoplasmic droplet, round cells in the ejaculation and defective sperm-head are the characteristics of objective assessment, which could be used for precocity selection of male sperm production. It can be concluded that the normality of sperm production in Guzerat breed, considering also all representative sperm production maturity process' parameters, and the scrotal circumference above $30 \mathrm{~cm}$, was reached over the age of 28 months.

Keywords: bulls; Guzerat; scrotal circumference; seminal traits; spermatic morphology. 


\section{Introdução}

Na seleção de animais para reprodução, além das características zootécnicas tais como peso corporal, qualidade de carcaça e tipo racial, deve-se dar ênfase à saúde reprodutiva dos animais, com avaliações clínicas que mostrem a normalidade dos órgãos reprodutivos e se há habilidade sexual (1). Dita seleção, visa atualmente não só a qualidade seminal, mas também a precocidade dos reprodutores. Pôr o estudo de características que possam estimar qualidade seminal em animais que ainda não atingiram a maturidade sexual pode predizer o futuro potencial reprodutivo dos mesmos.

Foi demostrado que a puberdade e maturidade sexual em machos, está intimamente ligada ao processo gradativo de liberação de gonadotrofinas e consequentemente da produção de testosterona $(2,3)$. Assim, a espermatogênese é iniciada quando ocorre um aumento na frequência de picos e na concentração da testosterona no plasma sanguíneo (3), que por sua vez, controla a diferenciação das células de Sertoli e, consequentemente, várias de suas funções (4). As gonadotrofinas (LH e FSH) controlam a secreção das células de Sertoli e Leydig desde a fase pós-natal, de modo que os esteróides e fatores de crescimento secretados por estas células têm ação direta ou indireta sobre o desenvolvimento das células germinativas. Este amadurecimento reflete na produção espermática pelo aumento gradativo da qualidade e concentração espermática, até atingir o equilíbrio em idade mais avançadas.

Desde esse ponto de vista, a capacidade reprodutiva dos touros, envolve vários parâmetros incluindo as medidas testiculares e a qualidade do sêmen (5). O perímetro escrotal (PE) é o parâmetro mais amplamente utilizado. Principalmente em função da facilidade de medição, e por estar diretamente relacionado às características qualitativas e quantitativas seminais (6) e hormonais. Além disso, têm sido relatado heritabilidade de moderada até alta para o PE (7).

Objetivou-se por meio do presente trabalho estudar a expressão de parâmetros reprodutivos de machos da raça Guzerá, criados no cerrado brasileiro a pasto com suplementação não volumosa na seca. Por meio de características como o PE e sua relação com a produção em quantidade e qualidade espermática no período da puberdade até os 36 meses.

\section{Material e métodos}

\section{A. Local do experimento}

O presente estudo foi realizado no município de Brasilândia, microrregião de Paracatu, ao noroeste do estado de Minas Gerais, região pertencente ao cerrado Brasileiro. Numa altitude de 550 metros em relação ao nível do mar. A coordenada geodésica da sede da propriedade é $17^{\circ} 032^{\prime} 54^{\prime \prime}$ de latitude sul e $46^{\circ} 13^{\prime} 227^{\prime \prime}$ de longitude Oeste. O clima é tropical chuvoso, com chuvas concentradas no período quente entre outubro e abril (mais de $90 \%$ do total anual). O inverno (junho a agosto) é muito seco, com precipitações totais mensais inferiores a $20 \mathrm{~mm}$. A temperatura média do mês mais frio (julho) é superior a $18{ }^{\circ} \mathrm{C}$ e as maiores temperaturas ocorrem geralmente em setembro, antecedendo o período chuvoso (8).

\section{B. Animais e tratamento}

Foram avaliados um total de 191 machos da raça Guzerá desde os 12 meses até os 36 meses de idade. Registrados como livro aberto (LA) pela Associação Brasileira de Criadores de Zebu (ABCZ). Após a desmama, os animais sob avaliação foram mantidos em pastagens Brachiarias brizantha e Andropogun gallanus suplementados com sal mineral sem uréia. Nos meses de maio e junho a suplementação mineral foi do tipo proteinado. Durante o período experimental, os machos foram mantidos em um lote único, contendo somente machos jovens, sem nenhum tipo de contato com fêmeas u outras categorias de animais. Os mesmos, foram submetidos a quatro avaliações anuais da biometria testicular e características seminais. Ditas avaliações foram feitas nos meses de janeiro (logo após o início das águas abril (final das águas), julho (no meio da seca) e outubro (final da seca). 


\section{Biometria testicular e avaliação das ca- racterísticas seminais}

As avaliações de biometria testicular consistiram na mensuração de comprimento e largura com auxílio de paquímetro, e PE obtido com auxílio de fita métrica na região mais larga do escroto, após leve tracionamento ventro-caudal das gônadas, segundo a técnica preconizada pelo Manual para Exame Andrológico e Avaliação de Sêmen Animal do (9).

As amostras de sêmen foram obtidas por eletro-ejaculação (Duboi ${ }^{\circledR}$ Eletroejaculador, Campo Grande - MS), Procurando-se, quando ocorreu turvação da amostra, manter a estimulação elétrica até o clareamento da mesma. Os animais que não respondiam ao primeiro estímulo eram imediatamente submetidos à outra tentativa. Logo após a coleta, foi realizado o exame das características físicas do ejaculado (motilidade, vigor e concentração espermática).

A avaliação da motilidade e vigor foram realizadas pela deposição de uma alíquota de sêmen entre lâmina e lamínula previamente aquecida a 37 ${ }^{\circ} \mathrm{C}$ e levadas à microscopia de luz com aumento de 100 a 400x. A motilidade foi expressa como o percentual de espermatozoides móveis. O vigor, representado pela força do movimento e a velocidade dos espermatozoides, foi classificado numa escala de zero a cinco onde zero caracteriza a ausência de movimento e cinco um movimento vigoroso e veloz dos espermatozoides segundo o CBRA (1998). A concentração espermática foi estimada indiretamente a partir do aspecto do ejaculado, o qual foi determinado subjetivamente pelo grau de turgidez da amostra categorizando-se em 0 ou transparente (ausência de espermatozoides); 1 ou aquoso ( $<200$ milhões de sptz/mL); 2 u opalescente (200-500.000 milhões de sptz/mL); 3 ou leitoso (500 milhões até 1 bilhão de sptz/mL) e 4 ou cremoso ( $>1$ bilhão de sptz/mL). A presença de células arredondadas no ejaculado foi avaliada no momento da avaliação da motilidade (11). A quantidade foi registrada seguindo uma escala de escore variando de 0 a 6 . $O$ valor zero foi usado na ausência deste tipo de células e 6 quando a proporção células arredondadas / espermatozoide foi superior a $0.5(50 \%)$ considerando a avaliação em pelo menos cinco campos visuais em microscopia de luz com aumento de 100 a 400x. Para a avaliação da morfologia espermática foram utilizadas alíquotas de sêmen preservadas em solução formol-salina tamponada (10), mantida resfriada a $5{ }^{\circ} \mathrm{C}$ até a avaliação. Para a avaliação, foi empregada a técnica de preparação úmida e análise de espermatozoides em microscopia de contraste de fase com aumento de 1000x (Microscopio Olimpus ${ }^{\circledR}$ BX41) onde foram avaliadas 200 células. Os defeitos espermáticos foram registrados seguindo a localização dos mesmos em defeitos de cabeça, peça intermediária, e peça principal.

\section{Puberdade}

A ocorrência da puberdade foi considerada a partir do momento em que o animal apresentou pelo menos um espermatozoide com motilidade progressiva, após coleta por eletro-ejaculação, segundo descrito por (11).

\section{E. Análise estatística}

Para a formação de arquivos, consistência e análise descritiva dos dados foram utilizados procedimentos contidos no pacote estatístico SAS (12). Para análise estatística dos dados foram calculadas as médias simples das características avaliadas e seus respectivos desvios-padrão. Foi utilizada estatística descritiva para caracterização das respostas avaliadas, como a identificação da cronologia do início da produção espermática. Foram realizadas regressões em função do PE com todos os parâmetros espermáticos, empregando-se o programa Sigma Plot versão 10.0, Copright ${ }^{\odot}$ - Systat Software, INC. 2006, para caracterização das respostas avaliadas. Foram também determinados os coeficientes de correlação de Pearson para respostas paramétricas como idade, $\mathrm{PE}$, e Spearman para respostas não paramétricas (motilidade, vigor, concentração, células arredondadas, e defeitos de cabeça), já para os defeitos de peça intermediaria e defeitos de peça principal, foram realizados a transformação angular para ajuste da normalidade empregando-se a função de Arco-seno da raiz, obtendo resposta paramétrica. Os coeficientes de correlação entre as variáveis de acordo com a norma- 
lidade de cada resposta foram avaliados segundo o método descrito por Sampaio (13).

\section{Resultados}

Após a detecção dos primeiros espermatozoides móveis, foi estudada a evolução das diferentes características físicas dos ejaculados dos machos. Dessa forma, observou-se aumento gradativo do PE com a idade. Com isso, foram constatadas altas correlações do PE de touros da raça Guzerá com idade $(r=0.97)$, motilidade $(r=0.94)$, vigor $(r=1.00)$ e concentração espermática $(r=0.94)$ $(P<0.0001)$. A frequência de defeitos espermáti- cos e consequentemente de espermatozoides normais por faixa etária estão apresentados na Tabela 1 , onde se verifica diminuição através da idade para os defeitos espermáticos relacionados a cabeça e acrossoma. De forma que, foi constatada uma relação alta e negativa entre eles $(r=-0.94$; $\mathrm{P}<0.0001)$. Para os defeitos relacionados à peça intermediaria, verifica-se alta incidência degota citoplasmática proximal nas diferentes faixas etárias, principalmente entre 12 e 16 meses (Chegando a atingir $48 \%$ do ejaculado). Para estes defeitos, também foi constatada diminuição deles através da idade, com correlação alta e negativa $(r=-0.98$; $\mathrm{P}<0.0001)$.

Tabela I. Perímetro escrotal (PE) e Frequências dos defeitos morfológicos (em porcentagem) nas diferentes faixas etárias de touros jovens da raça Guzerá criados no cerrado mineiro.

\begin{tabular}{|c|c|c|c|c|c|c|}
\hline \multicolumn{3}{|c|}{ Variáveis } & \multirow{2}{*}{$\begin{array}{c}\text { Categoria } \\
\text { (meses) } \\
20-24\end{array}$} & \multirow[b]{2}{*}{ 24-28 } & \multirow[b]{2}{*}{ 28-32 } & \multirow[b]{2}{*}{$32-36$} \\
\hline & $12-16$ & $16-20$ & & & & \\
\hline$P E$ & $16,1 \pm 1,5$ & $18,0 \pm 2,5$ & $21,2 \pm 2,9$ & $25,7 \pm 4,1$ & $28,4 \pm 3,3$ & $30,2 \pm 3,4$ \\
\hline Delgado & 0,86 & 0,69 & 1,98 & 1,61 & 2,53 & 2,49 \\
\hline Piriforme & 9,29 & 3,31 & 0,69 & 0,80 & 0,51 & 0,27 \\
\hline Pequeno & 0,14 & 0,00 & 0,38 & 0,18 & 0,02 & 0,00 \\
\hline Gigante & 0,00 & 0,00 & 0,00 & 0,04 & 0,00 & 0,04 \\
\hline Cabeça Sub & 0,29 & 0,92 & 0,26 & 0,09 & 0,15 & 0,00 \\
\hline Cabeça Isolada & 2,14 & 4,44 & 2,02 & 1,39 & 1,10 & 1,35 \\
\hline Acrossoma & 4,43 & 2,22 & 0,26 & 0,36 & 0,19 & 0,02 \\
\hline Cabeça Anormal & 0,86 & 0,61 & 0,69 & 0,36 & 1,02 & 0,55 \\
\hline Cabeça Dupla & 0,00 & 0,00 & 0,00 & 0,00 & 0,00 & 0,00 \\
\hline Retroaxial & 0,29 & 0,03 & 0,14 & 0,39 & 0,69 & 0,20 \\
\hline Abaxial & 1,29 & 1,42 & 0,57 & 0,25 & 0,24 & 0,14 \\
\hline$P I I r r$ & 15,86 & 13,89 & 2,50 & 0,58 & 0,61 & 0,12 \\
\hline PI Dobrada & 2,00 & 2,58 & 2,50 & 1,91 & 1,48 & 0,61 \\
\hline GCP & 48,14 & 41,42 & 28,10 & 15,95 & 3,92 & 3,43 \\
\hline$G C D$ & 0,29 & 1,06 & 1,79 & 1,63 & 1,38 & 1,80 \\
\hline PP Dobrada & 0,14 & 1,22 & 4,17 & 2,24 & 1,00 & 1,08 \\
\hline PP Enrolada & 0,00 & 1,97 & 4,83 & 1,78 & 0,71 & 0,80 \\
\hline Cabeça DGCP & 4,86 & 0,92 & 0,33 & 0,22 & 0,52 & 0,00 \\
\hline SPTZ Nor & 15,71 & 24,00 & 46,98 & 68,81 & 82,89 & 87,02 \\
\hline
\end{tabular}

Cabeça Sub: Cabeça subdesenvolvida; PI: Peça intermediaria; GCP: Gota Citoplasmática Proximal; GCD: Gota Citoplasmática Distal; PP: Peça Principal; Cabeça DGCP: Cabeça Delgada com Gota Citoplasmática Proximal; SPTZ Nor: Espermatozoides Normais. 
Foi observada a presença de células arredondadas em 129 animais $(67.53 \%)$. A presença delas no ejaculado coincidiu com a detecção das primeiras células espermáticas e em múltiplas ocasiões até em tentativas de coletas precedentes à identificação de células espermáticas. Com o incremento da produção espermática observou-se uma queda gradativa das células arredondadas estando ausente na maioria dos ejaculados de machos mais próximos à maturidade sexual. Porém, não foram observadas associações com as variáveis estudadas.

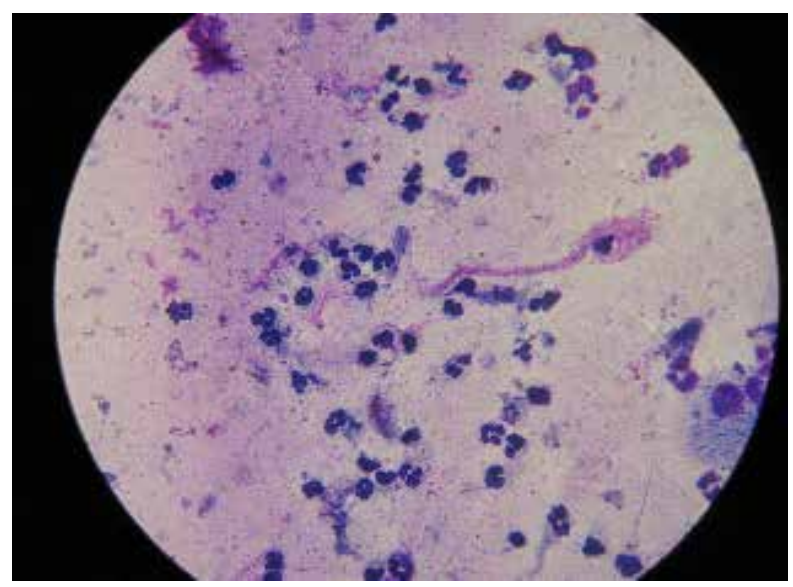

Fig. 1. Células arredondadas (polimorfo nucleares) presentes no ejaculado de macho da raça Guzerá na fase pré-puberal. Setas indicam a presença destes tipos celulares corados com a técnica de $\mathrm{H} \& \mathrm{E}$.

Verifica-se no último grupo (28 - 32 meses) a diminuição e queda na percentagem de defeitos espermáticos, encontrando-se por baixo dos $30 \%$ de defeitos totais, preconizado pelo (9).

\section{Discussão}

Bergmann (14) relataram que o PE está positivamente associado às características do sêmen como volume, vigor, concentração e motilidade dos espermatozoides. No presente trabalho, constatasse que a partir dos 12 meses (atingida a puberdade), e com o transcorrer do tempo, até os 28-36 meses de idade, a motilidade, o vigor e a concentração espermática aumentaram gradativamente. Confirmando os achados, mas antigos na literatura como
Wolf et al. (15) como os mais recentes $(16,17$, 18). Não entanto, valores de motilidade espermática aceitáveis são atingidos após a puberdade na faixa de idade de 9 aos 12 meses de idade para Bos taurus taurus, (19) e entre os 20 a 25 meses em Bos taurus indicus $(16,17,18)$.

A produção espermática e os parâmetros espermáticos aceitáveis pelo CBRA (9) 1acontecem de maneira geral mais tardiamente nos animais zebuínos (12 e 20 meses) quando comparadas com animais taurinos (6 e 9 meses de idade) segundo Hafez e Hafez B, Hafez (20) e os trabalhos de Frenau et al. (21) e Jimenez-Severiano (19). Estes acontecimentos, poderiam ser explicados por um amadurecimento mais tardio do eixo hipotalâmico-hipofisários-gônadas nos zebuínos (22). Sabe-se também que a espermatogênese é um evento extremamente delicado e que qualquer fator capaz de alterar esse processo resulta na produção de espermatozoides anormais (23), cabe ressaltar que, animais taurinos que atingem a puberdade com 6 meses de idade, são criados em condições favoráveis para isso acontecer. E no sentido contrário, de forma geral, animais zebuínos são criados no Brasil de forma extensiva sub o efeito ambiental e nutricional.

O PE é um importante parâmetro utilizado na avaliação do potencial reprodutivo de touros adultos, por apresentar correlações com determinadas características seminais (24). Diversos estudos têm sido feitos relacionando as medidas de PE com as características da qualidade do sêmen. Dentre eles, especificamente o trabalho de Quirino (25), demonstrou a utilização da medida do PE como ferramenta de seleção indireta de reprodutores bovinos, observando ser uma medida de correlação favorável com qualidade seminal. Hahn et al. (26) verificaram correlação positiva entre a mensuração testicular e a concentração espermática $(r=0.81)$ em touros da raça Holandesa, sendo esta correlação mais alta nos animais jovens (1 a 2 anos), tendendo a diminuir $(r=0.22)$ com a idade $(6$ a 12 anos). No presente trabalho foi constatada ditos resultados, de forma que a motilidade, vigor e concentração espermática aumentaram gradativamente e de forma linear com o tamanho do PE apre- 
sentando correlações altas e positivas $(r=0.94$; $r=1.00$ e $r=0.94$ respectivamente; $P<0.0001)$.

Dessa forma, a concentração espermática apresentou valores crescentes até a última categoria de idade avaliada, sugerindo que o platô da produção espermática poderia ainda não ter sido atingida aos 36 meses. Esta sugestão, no entanto, tem que ser avaliada com reserva haja vista o método de coleta de sêmen utilizado (eletro-ejaculação). Pois dependendo da duração do estímulo, pode mudar a concentração espermática de um ejaculado que somada à metodologia subjetiva de avaliação da concentração espermática utilizada não permitiu uma avaliação mais precisa deste parâmetro. Cabe ressaltar de igual forma, que a padronização desta última variável é muito complexa quando se trabalha com levantamentos feitos em propriedades particulares.

Adicionalmente, trabalhos como o de Bertschinger et al. (27) observaram em animais aos 18 meses de idade, uma relação curvilínea entre o PE e a morfologia espermática $(P<0.001)$. Sendo que testículos com perímetros escrotais de $38 \pm 1 \mathrm{~cm}$ produziram sêmen com altas taxas (80\%) de espermatozoides normais. Defeitos de cabeça geralmente tem alta incidência no início do processo espermatogênico quando comparado aos desejados em reprodutores adultos. Não entanto, dita incidência destes na população de machos avaliados apresentou valores próximos do desejado $(<10 \%)$ a partir dos 24 $\mathrm{cm}$ de PE.

A incidência de defeitos de peça intermediária também decaiu de forma linear na faixa de PE analisada. No entanto, apesar dos defeitos de peça intermediária decair gradativamente com ao aumento do PE os índices alcançados no pico da ocorrência não alcançaram uma faixa de valores que potencialmente poderia interferir na fertilidade dos machos. O mesmo raciocínio pode ser aplicado à incidência de defeitos de peça principal no ejaculado que foi caracterizada por uma equação $\widehat{\hat{Y}}=-$ $84.13+7.26 \mathrm{X}-0.1452 \mathrm{X}^{2}\left(\mathrm{R}^{2}=0.76\right)(\mathrm{P}<0.001)$. Os maiores valores para esta característica ocorreram na faixa de PE de 24 aos 28 meses, regredindo gradativamente com perímetros escrotais maiores.
Portanto, a incidência de defeitos da cabeça do espermatozoide e da incidência de gota em função de uma idade determinada pelo PE poderia ser utilizada como parâmetros para analisar a precocidade ou não do processo de equilíbrio do processo espermatogênico e de maturação espermática no epidídimo. A faixa de PE entre 24 e $26 \mathrm{~cm}$ poderia ser usada para estas duas características espermáticas para comparar o grau de maturidade da produção espermática entre contemporâneos.

Os animais apresentaram no período próximo à pós-puberdade a incidência de defeitos espermáticos elevados. Principalmente pela alta incidência de gota citoplasmática proximal. Foram observados também, porém, em menor proporção, defeitos de cabeça piriforme, cabeça isolada e defeitos de acrossoma. A diminuição destes defeitos com o aumento de idade, indicam que os processos de espermatogêneses precisam de um certo tempo após o início da puberdade para se ajustar e entrar em equilíbrio, provavelmente pela regulação do eixo hipotálamo-hipófise-gônadas e a sensibilidades deles aos hormônios produzidos.

O elevado percentual de gota citoplasmática no ejaculado de animais pós-púberes, também foi relatado por outros autores como Frenau et al. (21) e Carmo et al. (17). Isso, pode ser indicativo de deficiência no processo de maturação espermática que ocorre no epidídimo. Este defeito foi o último em atingir níveis considerados aceitáveis comparados aos demais Defeitos morfológicas dos espermatozoides, sugerindo que o equilíbrio do processo espermatogênico precede o equilíbrio e processo de maturação espermática que ocorre a nível de epidídimo.

O processo de maturação espermática atingiu sua plenitude entre os 24 e 28 meses na grande maioria dos animais. Enquanto as demais patologias associadas à puberdade atingiram frequências basais em categorias de idades inferiores. Sendo assim, estes parâmetros podem ser utilizados em conjunto, como referência para seleção de animais de maior precocidade quanto à morfologia espermática. A partir desta faixa de idade (24-28 meses), a incidência total de defeitos espermáticos encontradas no presente estudo foram observadas dentro 
dos limites preconizados pelo CBRA (9); < 30\% de defeitos morfológicos totais.

Foi observado em alguns machos nas diferentes fases peri-puberais a presença de células arredondadas no ejaculado. A mesma ocorreu, em alguns casos, inclusive antes da detecção das células espermáticas. As células foram identificadas com sendo polimorfos nucleares (células de defesa) neutrófilos (Figura 1). Em humanos e em animais a presença deste tipo celular foi associada a processos inflamatórios de partes dos órgãos genitais (28). Porém, no presente trabalho, não foi associado à presença delas a qualquer tipo de processo inflamatório em algum segmento dos órgãos genitais.

Não foi encontrado na literatura qualquer referência à presença deste tipo celular associado ao processo de puberdade em animais de origem zebuíno. Por ser sua presença transitória e não sendo as mesmas identificadas em idades mais avançadas ou com perímetros escrotais maiores do que $32 \mathrm{~cm}$ é possível especular que por sua característica transitória, a sua incidência está associada ao processo da puberdade.

Poderia se associar também ao equilíbrio do sistema genital à presença de espermatozoides nos ductos do sistema genital por onde transitam as células espermáticas. Sendo que, as células espermáticas, possuem um número de cromossomas diferentes ao das células somáticas do organismo e são consideradas estranhas ao mesmo. Podendo caracterizar o incremento temporário deste tipo celular uma resposta de ajuste do organismo, particularmente do sistema genital ao transito de células consideradas "estranhas".

Não foi possível realizar um estudo mais aprofundado do local de origem deste tipo de células, o que poderia auxiliar no entendimento dos mecanismos e na interpretação do significado da presença das mesmas. É esperado, baseando-se nas observações do presente experimento, que ejaculados de animais com PE acima dos $28 \mathrm{~cm}$ deveriam ter poucos ou até estarem livres da presença destas células.
Na população analisada a partir da categoria de idade de 24 aos 28 meses foi observado que os valores de motilidade e vigor espermáticos atingiram um patamar. Ditos valores considerados aceitáveis para motilidade, vigor e concentração foram alcançados quando o PE está próximo dos $32 \mathrm{~cm}$. Faixa na qual foi observada uma queda linear na incidência de defeitos de cabeça, peça intermediária e peça principal considerado adequado para reprodutores (9). Não entanto, a tendência da curva da concentração espermática $(r=1.0)$ sugere que o platô aos 36 meses de idade ainda não tinha sido atingido. No trabalho de Taylor et al. (29) em raças taurinas o platô da concentração espermática foi atingido entre os 20 aos 22 meses de idade. Enquanto que nas raças zebuínas o mesmo foi atingido com idade de 40 meses segundo o trabalho de Quirino et al. (25).

Palasz et al. (30) verificaram em touros Bos taurus taurus, correlação do PE com a produção diária de espermatozoides. Sendo que animais de PE maior que $31 \mathrm{~cm}$ apresentaram maior produção espermática que os animais com perímetro menor que $30 \mathrm{~cm}$. Dito de outra forma, e levando em consideração os achados no presente estudo, animais maturos sexualmente com PE acima da média, atingiriam os critérios de motilidade, vigor e concentração de igual forma que animais com PE dentro da média, diferindo unicamente na quantidade da produção diária de espermatozoides.

Já no caso de animais nas faixas peri-puberais e pós-puberdade, foi observado uma alta correlação do PE com idade, concentração, motilidade, vigor e defeitos espermáticos.

\section{Conclusão}

As características de motilidade, vigor, concentração espermáticas, anormalidade de cabeça de espermatozoide, peça intermediária e presença de gotas citoplasmáticas e a presença de células arredondadas no ejaculado foram características associadas ao processo de maturação da produção espermática posterior a ocorrência da puberdade.

As mesmas, variaram com a idade e o perímetro escrotal no período próximo pós-puberal. 
Pela ordem, a presenças de espermatozoides com gota citoplasmática proximal, células arredondadas no ejaculado e defeitos de cabeça dos espermatozoides são as características de avaliação objetiva que mais poderiam ser utilizados para seleção de precocidade da produção espermática em machos bovinos.

O perímetro escrotal pode ser utilizado como parâmetro exclusivo de seleção de reprodutores jovens da raça Guzerá, considerando como ponto de referência mais de $30 \mathrm{~cm}$. Sendo que, a normalidade da produção espermática na raça Guzerá considerando conjuntamente todos os parâmetros representativos de maturidade do processo da produção espermática aconteceram no presente trabalho, acima deste valor.

\section{Referencias}

(1) Corrêa AB, Vale Filho V, Corrêa GS, Andrade VJ, Silva MA, Dias JC. Caracteristicas do sêmen e maturidade sexual de touros jovens da raça Tabapuã (Bostaurus indicus) em diferentes manejos alimentares. Arq. Bras. Med. Vet. Zootec. 2006; 58(5): 823-830.

(2) Brito LFC. Nutrition, metabolic hormones, and sexual development in bulls. 179f. Doutorado - University of Saskatchewan, Saskatoon; 2006.

(3) Rawlins N, Evans ACO, Chandolia RK, Bagu ET. Sexual maturation in the bull. Reprod Dom Anim. 2008; 43(2): 295-301. DOI: http://dx. doi.org/10.1111/j.1439-0531.2008.01177.x.

(4) Griffin JE. Male reproductive funtion. In: Griffin JE, Ojeda SR (Eds.), Textbook of endocrine physiology. New York: Oxford University Press; 1988. pp. 165-185.

(5) Silva AEDF, Dode MAN, Unanian MM. Capacidade reprodutiva do touro de corte: funções, anormalidades e outros fatores que a influenciam. Campo Grande: EMBRAPA-CNPGC. 128p. (EMBRAPA-CNPGC. Documentos, 51); 1993.
(6) Lunstra DD, Ford JJ, Echterkamp SE. Puberty in beef bulls: Hormone cocentration, growth, testicular development, sperm production and sexual aggressiveness in bulls of different breeds. J. Anim. Sci. 1978; 46: 1054-1062.

(7) Oliveira MM, Rota EL, Dionello NJ, Aita MF. Herdabilidade e correlações genéticas do perímetro escrotal e idade ao primeiro parto com características produtivas em bovinos de corte; Revisão. R. Brás. Agrociencia, Pelotas. 2007; 13: 141-146.

(8) Agroambiental Rima (Relatorio Meio Ambiental). Fazendas reunidas. 2004.

(9) CBRA 1998. Manual para exame andrológico e avaliação de sêmen animal, 2 ed. Belo Horizonte: CBRA, 49p.:il.

(10) Hancock J. The morphology of boar spermatozoa. J. Roy. Micro. 1957. Soc.76: 84-97.

(11) Garcia JM, Pinheiro LEL, Okuda HT. Body development and semen physical and morphological caracteristics of young Guzera bulls. Ars. Vet. 1987; 3: 47-53.

(12) SAS. SAS/STAT User's Guide. Version 6.12. Statistical Analysis System Institute. Cary, NC, USA; 1996.

(13) Sampaio IBM. Estatística aplicada à experimentação animal. 2a. ed. Belo Horizonte: Fundação de Estudo e Pesquisa em Medicina Veterinária e Zootecnia; 2002.

(14) Bergmann JAG, Qwirino CR, Vale Filho VR, Andrade VJ, Fonseca CG. Herdabilidades e correlações genéticas entre medições testiculares e características espermáticas em touros Nelore. Arch. Latinoam. Prod. Anim. 1997; 5: 473-475.

(15) Wolf FR, Alquimist JO, Hale EB. Prepuberal behavior and pubertal characteristics of beef bulls on high nutrient allowance. J. Anim. Sci. 1965; 24: 761-764. 
(16) Ramírez López CJ, Rugeles Pinto CC, Castaño Villadiego FA, Gómez León VE, Miranda Neto T, Guimarães JD. Estadio de madurez sexual en toros de la raza nelore. Rev Med Vet. 2106; 31: 9-20.

(17) Carmo AS, Osório JP, Gomes RS. Parâmetros do desenvolvimento de machos Guzerá criados na região do cerrado a pasto com suplementação volumosa na seca. In: Congresso Brasileiro de Reprodução Animal, 17, Curitiba, PR, Anais...; 2007. p. 48.

(18) Dias JC. Comportamento sexual, concentrações periféricas de testosterona e perfil cromatográfico de proteínas do sêmen com afinidade a heparina, em touros jovens da raça Guzerá. 110 p. Doutorado - Escola de Veterinária, UFMG. Belo Horizonte; 2008.

(19) Jimenéz-Severiano H. Sexual development of dairy bulls in the Mexican tropics. Theriogenology. 2002, 58: 921-932.

(20) Hafez E, Hafez B. Reprodução animal. 7.ed. Barueri: Manole; 2004.

(21) Freneau GE, Vale Filho VR, Marques Jr. AP, Maria WS. Puberdade em touros Nelore criados a pasto no Brasil: características corporais, testiculares, seminais e índice de capacidade andrológica por pontos (ICAP). Arq. Bras. Méd. Vet. Zootec. 2006; 58(6): 1107-1115.

(22) Brito LF, Silva AE, Unanian MM, Dode MA, Barbosa RT, Kastelic JP. Sexual development in early- and late-maturing Bos indicus and Bos indicus $x$ Bos taurus crossbred bulls in Brazil. Theriogenology. 2004; 62: 1198-1217. DOI: http://dx.doi.org/10.1016/j.theriogenology.2004.01.006.

(23) Garcia AR. Efeitos do estresse térmico testicular e do uso da somatotropina recombinante bovina nas características seminais, integridade de membranas, função mitocondrial e estrutura da cromatina de espermatozoides de touros Simental (Bos taurus taurus). Tese (Doutorado) - Faculdade de Medicina Veterinária e Zootecnia, Universidade de São Paulo, Pirassununga, SP. 2004.

(24) Gilardi SGT, Pinho TG, Martins CB. Perímetro escrotale características do sêmen de touros Nelore aos 18 meses de idade. Rev. Bras. Ci. Vet. 2001; 8(1):13-15.

(25) Quirino CR, Bergmann JA, Vale Filho VR, Andrade VJ, Pereira JC. Evaluation of four mathematical functions to describe scrotal circumference maturation in Nelore bulls. Theriogenology. 1999; 52: 25-34. DOI: http:// dx.doi.org/10.1016/S0093-691X(99)00107-7.

(26) Hahn J, Foote RH, Seidel GE. Quality and freezability of semen from growing and aged dairy bulls. J. Dairy Sci. 1969; 52 (1): 18-43.

(27) Bertschinger HJ, Ehret WJ, Wood R. Beef bull performance, scrotal circumference and semen quality. In: International Congress on Animal Reproduction, 12, The Hague. Proceedings... The Hague: University of Waageningen 1992, p. 1525-1527

(28) Johanisson E, Campana A, Luthi R, Agostini ADE. Evaluation of round cells in semen analysis: a comparative study. Human Reproduction Update. 2000; 6(4): 404-412. DOI: http://dx.doi.org/10.1093/humupd/6.4.404.

(29) Taylor JF, Bean B, Marshall CE. Genetic and environmental components of semen production traits of artificial insemination Holstein Bulls. J. Dairy Sci. 1985; 68: 2703-2722. DOI: http://dx.doi.org/10.3168/jds.S00220302(85)81155-3.

(30) Palasz AT, Cates WF, Barth AD, Mapletoft RJ. The relationship between scrotal circumference and quantitative testicular traits in yearling beef bulls. Theriogenology. 1994; 42 (8): 715-726. DOI: http://dx.doi.org/10.1016/0093-691X(94)90388-Y. 